onset of symptoms. Thus iTreg have significant positive effects on PIL, which may have consequences for future approaches in treating SLE.

Disclosure of Interest: None declared

DOI: 10.1136/annrheumdis-2018-eular.6090

\section{FRI0260 POLYMORPHISMS OF STAT4 AND MIR146A PREDICT THE ACHIEVEMENT OF 5 YEARS REMISSION IN PATIENTS WITH SYSTEMIC LUPUS ERYTHEMATOSUS}

C. Perricone ${ }^{1}$, C. Ciccacci ${ }^{2}$, F. Ceccarelli ${ }^{1}$, G. Mettola ${ }^{1}$, I. Leccese ${ }^{1}$, F.R. Spinelli ${ }^{1}$, C. Alessandri ${ }^{1}$, C. Politi ${ }^{2}$, A. Latini ${ }^{2}$, G. Novelli ${ }^{2}$, G. Valesini ${ }^{1}$, P. Borgiani ${ }^{2}$, F. Conti ${ }^{1} .{ }^{1}$ Lupus Clinic, Rheumatology, Department of Internal Medicine, Sapienza University of Rome; ${ }^{2}$ Department of Biomedicine and Prevention, Section of Genetics, University of Rome "Tor Vergata", Rome, Italy

Background: Systemic lupus erythematosus (SLE) is a chronic autoimmune disease with a complex pathogenesis in which genes and environmental factors interact leading to a protean clinical picture. Treat-to-target recommendations have identified 'remission' as a target in SLE, since achievement of remission improves the outcome and is associated with decreased damage progression. Nonetheless, predicting factors for the achievement of remission are lacking. It is likely that genes associated with SLE pathogenesis may influence the disease course.

Objectives: Thus, our aim was to analyse previously identified loci associated with SLE in a cohort of SLE patients to evaluate their influence on remission achievement.

Methods: We recruited 117 Italian SLE patients. A panel of 34 SNPs in 19 genes involved in immune response, autophagy and inflammation, was selected. SNPs genotyping was performed by allelic discrimination assay by TaqMan assays (Applied Biosystems, Foster City, CA, USA) and ABI PRISM 7000. The main clini$\mathrm{cal} /$ laboratory features (including injury index and disease activity) were collected on an electronic platform. Remission was defined according to Zen et al. ${ }^{1}$ and evaluated over 5 years. A genotype/phenotype correlation analysis was performed.

Results: The variant alleles of rs7574965 (STAT4) $(p<0.001)$ and rs2910164 (MIR146a) $(p=0.031)$ were significantly associated with lack of achievement of 5 years remission in SLE. Specifically, patients carrying the C allele of MIR146a were less likely to achieve 5 years remission ( $p=0.01$, OR $0.235,95 \% \mathrm{Cl} 0.074-$ 0.752 ) as well as to achieve remission after 1,2 and 3 years of evaluation $(p=0.002, p=0.001, p=0.002$, respectively). Among the clinical and laboratory features, 5 years remission was less likely to be achieved by patients who had arthritis in their clinical history $(p=0.007)$, and who tested positive for anti-dsDNA $(p=0.005)$. In a multinomial logistic regression analysis, arthritis $(p=0.022, \operatorname{Exp}(B)$ $=0.255,95 \% \mathrm{Cl} 0.079-0.820)$, anti-dsDNA $(p=0.003, \operatorname{Exp}(B)=0.166,95 \% \mathrm{Cl}$ $0.051-0.537)$ and MIR146a rs2910164 gene variant $(p=0.046, \operatorname{Exp}(B)=0.250$, $95 \% \mathrm{Cl} 0.064-0.974$ ) were confirmed to be independent risk factors for unreached 5 years remission (table 1 ).

Abstract FRI0260 - Table 1

\begin{tabular}{lcccc}
\hline REMISSION & $\begin{array}{c}\text { Exp } \\
\text { (B) }\end{array}$ & & Exp (B) 95\% Cl \\
\hline & & & Lower & Upper \\
& Arthritis & 0255 & 0079 & 0,82 \\
anti- & 0166 & 0051 & 0537 \\
dsDNA & & & \\
STAT4 & 0354 & 0,11 & 1139 \\
& mir146A & 0,25 & 0064 & 0974 \\
\hline
\end{tabular}

Conclusions: We describe for the first time the contribution of STAT4 and MIR146a SNPs as predicting factors for the achievement of 5 years remission in SLE. No genetic study has been performed so far in SLE, while a genetic profile of patients may be useful to predict the disease outcome.

REFERENCE:

[1] Zen, et al. Ann Rheum Dis. 2017 Mar;76(3):562-565.

Disclosure of Interest: None declared

DOI: 10.1136/annrheumdis-2018-eular.6936

\section{FRI0261}

ASSESSMENT OF AUTOPHAGE FUNCTION IN SYSTEMIC LUPUS ERYTHEMATOSUS IN RESPECT OF HYPERLIPIDEMIA AND IMMUNOSUPPRESSIVE DRUGS

A.S. Hamada ${ }^{1}$, M.I. aref ${ }^{1}$, W.A. salahdin ${ }^{2} .{ }^{1}$ Clinical Immunology, Al Azhar University Hospital, Cairo; ${ }^{2}$ Rheumatology, Benha University Hospital, Benha, Egypt

Background: Autophagy is an orchestrated homeostatic process to eliminate unwanted proteins and damaged organelles. Lipid turnover, as well, is controlled by autophagy through a process described as lipophagy. Defective lipophagy has been already linked to important metabolic disorders such as fatty liver, obesity and atherosclerosis.

Objectives: Assessment of autophagy focusing on lipids regulation in untreated newly diagnosed systemic lupus Erythematosus (SLE) patients and after three months of treatment with immunosuppressive drugs.

Methods: Subjects in this study have been divided into three groups. Group 1 included 60 newly diagnosed SLE patients before receiving any treatment, group2 included the same subjects of group 1 after three months of treatment with immunosuppressive drugs and group 3 included 30 healthy donors of matched age and sex as a control group. For each subject, disease activity was assessed by (SLEDAI) score, lipid profile was measured in addition to evaluation of lipids uptake enhanced phagocytosis and intracellular killing ability of monocytes and neutrophils using Sudan Black B stain and Nitroblue tetrazolium stain mixed with latex particles coated with antibodies. Microscopic pictures were captured and quantified by ImageJ.

Results: $95 \%$ of patients were females $(57 / 60)$ with mean of age(39.7 \pm 8.6$)$. Mean of SLEDAI score in group 1 was $(18.6 \pm 3.4$ ) decreased in group 2 (3 months after treatment) to $(10.4 \pm 4.2)$. There was a positive correlation between total choles terol, LDL and triglycerides and disease activity(SLEDAI score) ( $r=0.677$, $r=0.603$ and $r=0.718$; respectively). On the contrary, There was a negative correlation between HDL and disease activity $(r=-0.396)$. Furthermore, there was a negative correlation between lipid content of cells and intracellular killing and disease activity $(r=-0.258$ and $r=-0.324$; respectively). After 3 months, $100 \%$ of patients were taking Corticosteroids and Hydroxychloroquine(60/60).18.3\% of patients received Azathioprine(11/60), 40.0\% received Cyclophosphamide(24/ 60 ) and $15 \%$ received Mycophenolate $(9 / 60)$ besides Corticosteroids and Hydroxychloroquine. Comparing group 2 to group 1, there was significant increase in cholesterol, $L D L$ and trigycerides $(p=0.027, p=0.021$ and $p=0.017$; respectively) while HDL showed insignificant difference $(p=0.0740)$. Lipid content in cells and intracellular killing significantly decreased $(p=0.0322$ and $p=0.0271$; respectively). Conclusions: Autophagy is deficient in patients with SLE aggravated by immunosuppressive drugs so they are more susceptible to infections and dyslipidemia. Consequently, lipid lowering drugs are definitely required to decrease comorbidity.

Disclosure of Interest: None declared

DOI: 10.1136/annrheumdis-2018-eular.4609

\section{FRI0262 MONITORING DISEASE ACTIVITY IN SYSTEMIC LUPUS ERYTHEMATOSUS WITH DIGITAL ELISA QUANTIFICATION OF SERUM INTERFERON-A}

S. Mouries-Martin ${ }^{1}$,A. Mathian ${ }^{1}$, K. Dorgham ${ }^{2}$, H. Devilliers ${ }^{3}$, F. Cohen-Aubart ${ }^{1}$, L. N. Garrido Castillo ${ }^{2}$, J. Haroche ${ }^{1}$, M. Hie ${ }^{1}$, M. Pineton De Chambrun ${ }^{1}$, M. Miyara ${ }^{1}$ M. Pha ${ }^{1}$, D. Lê Thi Huong ${ }^{1}$, F. Rozenberg ${ }^{4}$, G. Gorochov ${ }^{5}$, Z. Amoura ${ }^{1}$. ${ }^{1}$ French National Referral Center for Systemic Lupus Erythematosus, Antiphospholipid Antibody Syndrome and Other Autoimmune Disorders, Service de Médecine Interne 2, Institut E3M, Assistance Publique-Hôpitaux de Paris (APHP), Groupement Hospitalier Pitié-Salpêtrière; ${ }^{2}$ Inserm UMRS1135, Centre d'Immunologie et des Maladies Infectieuses (CIMI-Paris), Sorbonne Universités, UPMC Univ Paris 06, Paris; ${ }^{3}$ Centre Hospitalier Universitaire de Dijon, Hôpital François-Mitterrand, Service de Médecine Interne 2 et Centre d'Investigation Clinique, Inserm CIC 1432, DIJON; ${ }^{4}$ Service de Virologie, Assistance PubliqueHôpitaux de Paris (APHP), Hôpital Cochin; ${ }^{5}$ Inserm UMRS1135, Centre d'Immunologie et des Maladies Infectieuses (CIMI-Paris), Assistance PubliqueHôpitaux de Paris (APHP), Groupement Hospitalier Pitié-Salpêtrière, Paris, France

Background: To date, anti-dsDNA-Ab titration, better achieved with the Farr test, has been used to monitor global disease activity in systemic lupus erythematosus (SLE). Indeed, anti-DNA-Ab-positivity is associated with overall SLE activity. However, the sensitivity and specificity of that association are relatively low. The close association between Interferon alpha (IFN $\alpha$ ) expression and SLE activity suggests that monitoring this cytokine might help physicians better evaluate 